\title{
Metastatic Insulinoma Presenting as a Liver Cyst
}

\author{
Hua Li · Tony El Jabbour · Ankesh Nigam ${ }^{1} \cdot$ Hwajeong Lee \\ Departments of Pathology and Laboratory Medicine and ${ }^{1}$ General Surgery, Albany Medical Center, Albany, NY, USA
}

Insulinoma is usually detected early in the disease course as a benign form due to clinical manifestations associated with hyperinsulinism. ${ }^{1}$ Malignant insulinoma presenting as a hepatic cystic metastasis has not been described in the literature.

\section{CASE REPORT}

A 74-year-old woman with no significant past medical history presented with abdominal pain and vomiting. Abdominal computed tomography scan showed five enhancing hepatic masses measuring up to $7.6 \mathrm{~cm}$ in greatest dimension, and a cyst, measuring $6.2 \mathrm{~cm}$ in greatest dimension, in the medial segment of the left hepatic lobe (Fig. 1). One of the mass lesions was biopsied and showed a well-differentiated neuroendocrine tumor. Subsequent octreotide scan showed an additional focus of uptake in the pancreatic tail measuring $2.1 \mathrm{~cm}$.

The patient was placed under active surveillance. Three months later, she developed worsening diaphoretic episodes and tremors. Initial blood testing showed: proinsulin 1,290 pmol/L (range, 1.7 to $12 \mathrm{pmol} / \mathrm{L}$ ), glucose $42 \mathrm{mg} / \mathrm{dL}$ (range, 65 to 99 $\mathrm{mg} / \mathrm{dL}$ ), insulin $40.3 \mu \mathrm{IU} / \mathrm{mL}$ (range, 2.6 to $24.9 \mu \mathrm{IU} / \mathrm{mL}$ ), and C-peptide $10.4 \mathrm{ng} / \mathrm{mL}$ (range, 1.1 to $4.4 \mathrm{ng} / \mathrm{mL}$ ). Initial conservative management failed. Therefore, the patient underwent distal pancreatectomy with splenectomy and resection of the hepatic tumors and cyst.

Grossly, the pancreatic tail displayed a 2.6-cm-sized, ill-defined, firm and tan solid nodule. The resected hepatic segments

\section{Corresponding Author}

Hwajeong Lee, MD

Department of Pathology and Laboratory Medicine, Albany Medical Center, 47 New

Scotland Ave., MC81, Albany, NY 12208, USA

Tel: +1-518-2626903, Fax: +1-518-2623663,E-mail: LeeH5@amc.edu

Received: December 11, 2018 Revised: January 7, 2019

Accepted: January 14, 2019 exhibited multiple well-demarcated lobulated tan nodules, measuring up to $6.5 \mathrm{~cm}$ in greatest dimension. A portion of the hepatic cyst wall measuring $2.4 \mathrm{~cm}$ was also received.

Microscopic examination of the pancreatic tail nodule showed a proliferation of neoplastic cells arranged in nested and trabecular patterns (Fig. 2). The neoplastic cells were relatively uniform and cuboidal, with finely granular, amphophilic to eosinophilic cytoplasm and centrally located round to oval nuclei. A "salt and pepper" chromatin pattern was noted. The mitotic rate was less than 2 mitoses/10 high power fields and the Ki67 proliferation index was $0.05 \%$. A diagnosis of well-differentiated neuroendocrine tumor, World Health Organization (WHO) grade 1, was rendered. Given the clinical history, the overall findings were consistent with functioning pancreatic neuroendocrine tumor (PanNET), insulinoma. The liver masses were metastatic well-differentiated neuroendocrine tumor from the pancreas with the Ki67 proliferation index $1.75 \%$, WHO grade 1 .

The liver cyst was lined by neoplastic cells, which were morphologically identical with those in the pancreatic tumor. Scattered tumor nests were noted in the cyst wall. These cells were positive for synaptophysin and cytokeratin AE1/AE3, confirming the diagnosis of cystic metastasis of pancreatic well-differentiated neuroendocrine tumor (Fig. 3).

The postoperative course was uneventful. The postoperative insulin level dropped to normal. The patient has been free of symptoms for 3 months and is doing well.

This study was approved by the Institutional Review Board of Albany Medical Center with a waiver of informed consent (IRB Protocol \#5036, Date 1/16/2018) and performed in accordance with the principles of the Declaration of Helsinki. 


\section{DISCUSSION}

PanNETs, also known as islet cell tumors, account for $1 \%-2 \%$

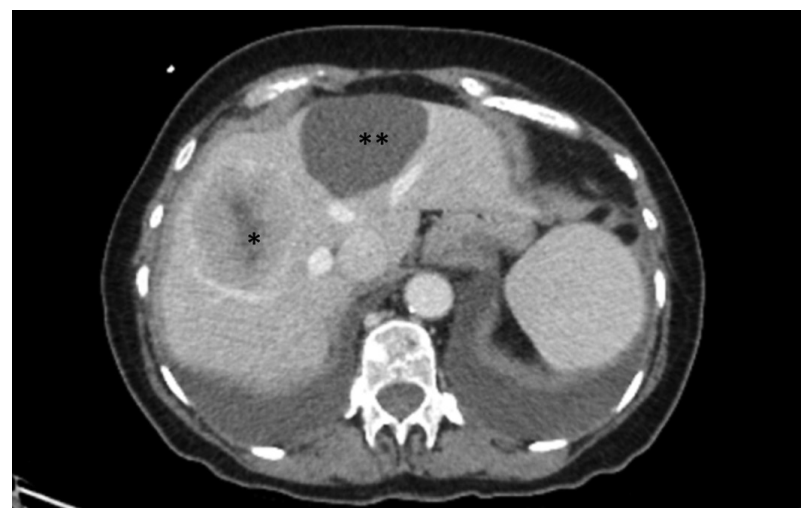

Fig. 1. Computed tomography shows one of the metastatic nodules $\left(^{*}\right)$ and a cyst $\left(^{(*)}\right.$ in the liver. of pancreatic tumors. ${ }^{2}$ PanNETs are classified as functioning or non-functioning depending on the secretion of bioactive peptides. Functioning PanNETs secrete one or more biologically active peptides such as insulin, gastrin, glucagon, somatostatin, and vasoactive intestinal peptide, and induce corresponding clinical symptoms, while non-functioning PanNETs are usually asymptomatic. ${ }^{2}$ Due to the clinical symptoms resulting from the secretion of biologically active peptides, functioning PanNETs tend to present earlier in the disease course, whereas non-functioning tumors remain silent until metastasis and associated symptoms ensue. $^{3}$

In about $40 \%-80 \%$ of patients with PanNETs, metastases are detected at diagnosis, commonly in the liver. ${ }^{4}$ Cystic PanNETs demonstrate indolent clinical behavior compared to solid tumors. ${ }^{5}$ Metastatic neuroendocrine tumors are usually solid; however, cystic hepatic metastases of ileal neuroendocrine tumor have been re-
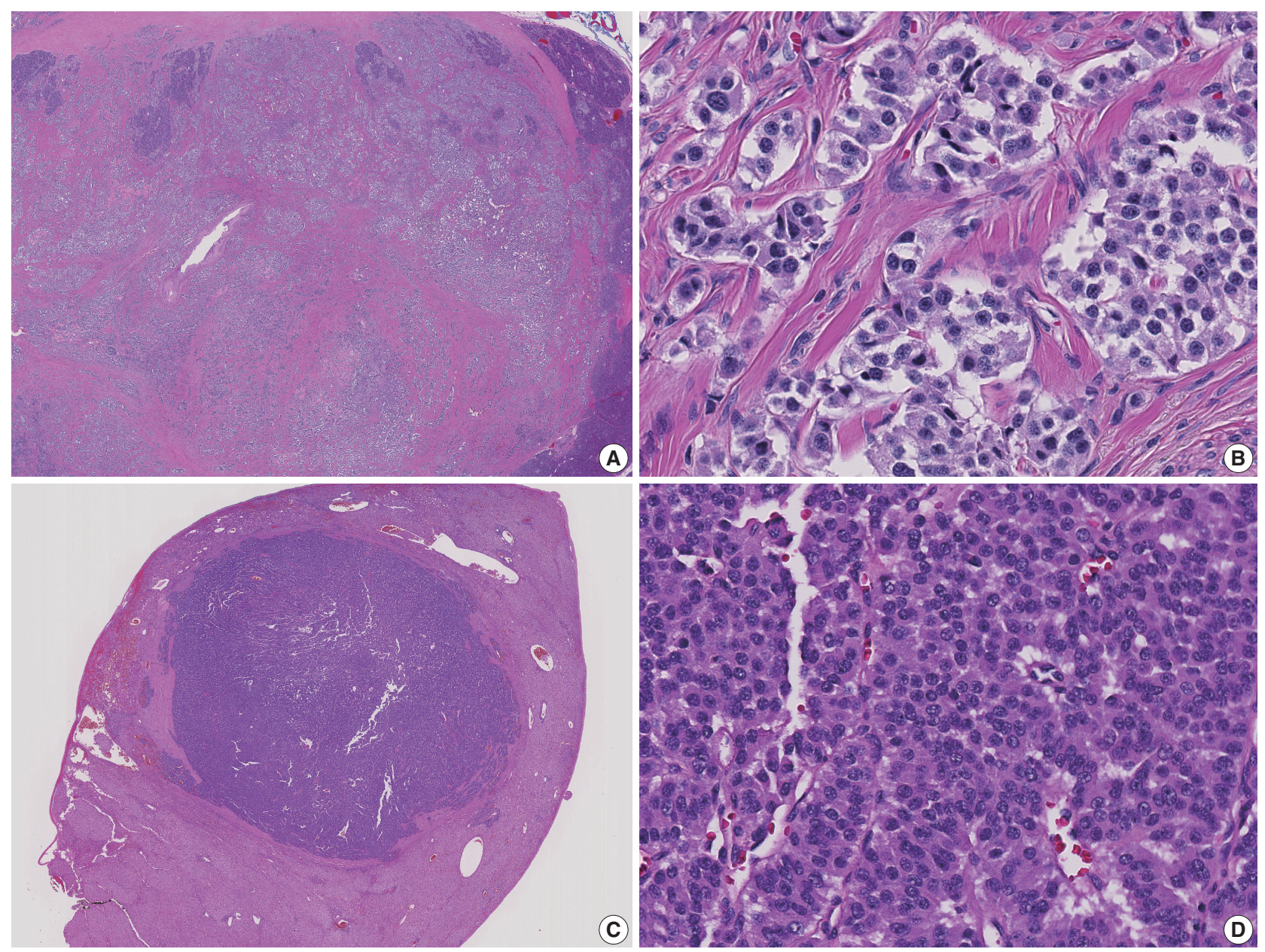

Fig. 2. Primary pancreatic neuroendocrine tumor and hepatic metastasis. (A) Scanning view of the tumor in the pancreas. (B) Higher magnification view shows "salt and pepper" chromatin pattern of the tumor cells. (C) Scanning view of the solid hepatic metastasis. (D) Higher magnification view of the hepatic metastasis shows trabecular growth pattern. 

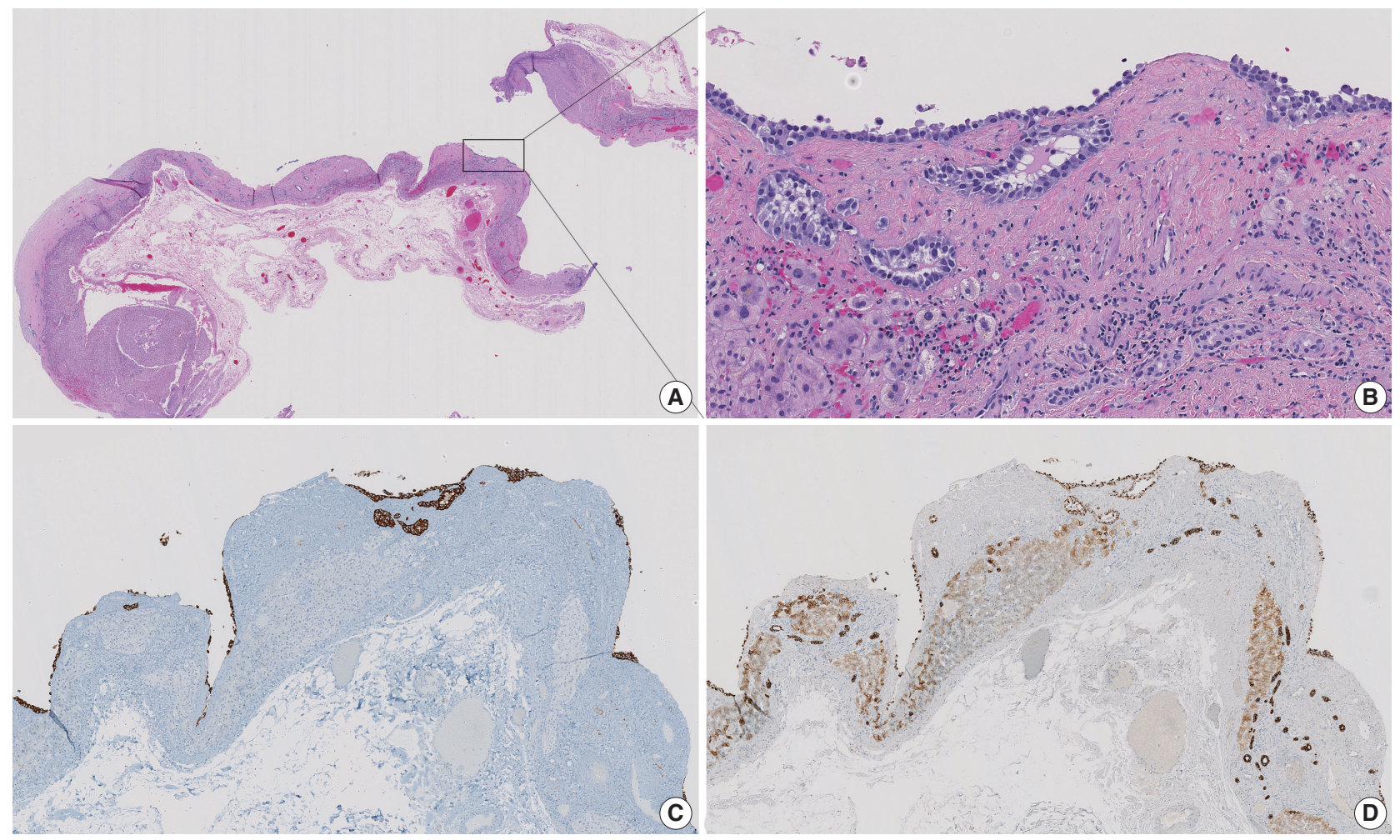

Fig. 3. Hepatic cystic metastasis of pancreatic well-differentiated neuroendocrine tumor. (A) Scanning view shows cyst walls. (B-D) Mediumpower view demonstrates neoplastic cells lining the cyst (B) that are positive for synaptophysin (C) and cytokeratin AE1/AE3 (D).

ported. ${ }^{6,7}$ Metastatic PanNET presenting as a liver cyst has not been reported in the literature.

Insulinoma is the most common functioning PanNET and constitutes 30\%-45\% of the cases. ${ }^{1}$ Insulinoma is usually smaller $(<2 \mathrm{~cm}$ in diameter) than non-functioning PanNETs (often larger than $5 \mathrm{~cm}$ in diameter), due to the early diagnosis of insulinoma secondary to symptoms associated with hyperinsulinism. ${ }^{2}$ Interestingly, however, symptoms related to hypoglycemia manifested late in the disease course in our patient, 3 months after the confirmation of liver metastasis. Our case illustrates that the tumor burden of functioning PanNET may not always correlate with the severity of the symptoms. Alternatively, a neuroendocrine tumor may acquire functionality during the disease progression and a nonfunctioning tumor may transition into a functioning tumor. ${ }^{8}$

Our objective is to present a unique case of cystic hepatic metastasis of a functioning PanNET. Moreover, metastatic insulinoma is rare (range, $2.4 \%$ to $17.9 \%$; average, $8.4 \%$ ). ${ }^{2,9}$ Cystic lesions of the liver encompass a variety of pathologic entities. Although rare, cystic metastasis of neuroendocrine tumor needs to be considered as a differential diagnosis when evaluating hepatic cysts. Notably, cystic metastasis of neuroendocrine tumor in the liver would be a diagnostic pitfall when the primary lesion is occult, as have often been described. ${ }^{10}$

\section{ORCID}

Hua Li: https://orcid.org/0000-0001-7481-3942

Tony El Jabbour: https://orcid.org/0000-0002-6823-1919

Ankesh Nigam: https://orcid.org/0000-0002-7565-6333

Hwajeong Lee: https://orcid.org/0000-0001-7005-6278

\section{Author Contributions}

Conceptualization: HL (Hwajeong Lee).

Data curation: HL (Hua Li).

Formal analysis: $\mathrm{HL}$ (Hua Li), TEJ.

Investigation: $\mathrm{HL}$ (Hua Li), TEJ, AN, HL (Hwajeong Lee).

Methodology: HL (Hua Li), TEJ, HL (Hwajeong Lee).

Project administration: HL (Hwajeong Lee).

Resources: AN, HL (Hwajeong Lee).

Supervision: HL (Hwajeong Lee).

Validation: AN, HL (Hwajeong Lee).

Visualization: HL (Hua Li), TEJ.

Writing—original draft: HL (Hua Li).

Writing—review \& editing: HL (Hua Li), TEJ, AN,

HL (Hwajeong Lee). 


\section{Conflicts of Interest}

The authors declare that they have no potential conflicts of interest.

\section{REFERENCES}

\section{Halfdanarson TR, Rubin J, Farnell MB, Grant CS, Petersen GM} Pancreatic endocrine neoplasms: epidemiology and prognosis of pancreatic endocrine tumors. Endocr Relat Cancer 2008; 15: 409-27.

2. Bosman FT, Carneiro F, Hruban RH, Theise ND. WHO classification of tumours of the digestive system. 4th ed. Lyon: International Agency for Research on Cancer, 2010; 322-6.

3. Halfdanarson TR, Rabe KG, Rubin J, Petersen GM. Pancreatic neuroendocrine tumors (PNETs): incidence, prognosis and recent trend toward improved survival. Ann Oncol 2008; 19: 1727-33.

4. Zhang X, Song J, Liu P, et al. A modified M-stage classification based on the metastatic patterns of pancreatic neuroendocrine neoplasms: a population-based study. BMC Endocr Disord 2018; 18: 73.

5. Cloyd JM, Kopecky KE, Norton JA, et al. Neuroendocrine tumors of the pancreas: degree of cystic component predicts prognosis. Surgery 2016; 160: 708-13.

6. Fiori S, Del Gobbo A, Gaudioso G, et al. Hepatic pseudocystic metastasis of well-differentiated ileal neuroendocrine tumor: a case report with review of the literature. Diagn Pathol 2013; 8: 148.

7. Isomura T, Kojiro M, Kawano Y, et al. Small multiple carcinoid tumors occurring in the ileum with a pseuodcystic liver metastasis. Acta Pathol Jpn 1980; 30: 137-43.

8. Yoshioka M, Shibata S, Uchinami $\mathrm{H}$, et al. The transformation of a nonfunctioning islet cell tumor of the pancreas into a proinsulinoma under conditions of lung metastasis. Intern Med 2015; 54: 785-90.

9. Bozkirli E, Bakiner $\mathrm{O}$, Abali $\mathrm{H}$, et al. A case of inoperable malignant insulinoma with resistant hypoglycemia who experienced the most significant clinical improvement with everolimus. Case Rep Endocrinol 2013; 2013: 636175.

10. Alexandraki K, Angelousi A, Boutzios G, Kyriakopoulos G, Rontogianni D, Kaltsas G. Management of neuroendocrine tumors of unknown primary. Rev Endocr Metab Disord 2017; 18: 423-31. 